\title{
Efficacy and safety of different molecular targeted agents based on chemotherapy for gastric cancer patients treatment: a network meta-analysis
}

\author{
Zheng Ren ${ }^{1, *}$, Jinping Sun ${ }^{1, *}$, Xinfang Sun ${ }^{1}$, Hongtao Hou ${ }^{1}, \mathrm{Ke} \mathrm{Li}^{1}$ and Quanxing $\mathbf{G e}^{1}$ \\ ${ }^{1}$ Department of Digestive Internal Medicine, Huaihe Hospital of Henan University, Kaifeng 475000, Henan, China \\ *These authors contributed equally to this work
}

Correspondence to: Quanxing Ge, email: hongluojiadoctor@163.com

Keywords: gastric cancer, molecular targeted agents, chemotherapy, network meta-analysis, efficacy

Received: February 09, $2017 \quad$ Accepted: March 23, $2017 \quad$ Published: April 18, 2017

Copyright: Ren et al. This is an open-access article distributed under the terms of the Creative Commons Attribution License 3.0 (CC BY

3.0), which permits unrestricted use, distribution, and reproduction in any medium, provided the original author and source are credited.

\section{ABSTRACT}

Increasing numbers of reports have been published to demonstrate that molecular targeted agents are able to improve the efficacy of chemotherapy in gastric cancer. This network meta-analysis aimed to evaluate the efficacy and safety of different molecular targeted agents, which were divided into six groups based on the targets including hepatocyte growth factor receptor (c-MET), vascular endothelial factor and its receptor (VEGF/VEGFR), human epidermal growth factor receptor 2 (HER2), epidermal growth factor receptor (EGFR), mammalian target of rapamycin (mTOR) and tyrosine kinase inhibitor (TKI). These six groups of targeted agents were evaluated for their efficacy outcomes measured by overall survival (OS), progression-free survival (PFS) and overall response rate (ORR). While their safety was measured 7 adverse events, including fatigue, anaemia, vomiting, neutropenia, thrombocytopenia, diarrhea and nausea. A total of 23 articles were included after extensive searching and strict inclusion, HER2 and VEGF(R) turned out to be the two most effective targeted drugs for their outstanding performance in OS and PFS. However, they were associated with severe adverse events, including fatigue, neutropenia and diarrhea. Therefore, they should be used with caution during their application. In conclusion, VEGF(R) and HER2 have the potential to be the optimal target agents for their survival efficacy, while the adverse events associated with them should be paid attention in application.

\section{INTRODUCTION}

Gastric cancer (GC), also known as stomach cancer, is a malignant tumor caused by environmental and genetic factors. Although the incidence and mortality of GC have extremely decreased since the 21 st century, it is still the fourth major cause of cancer deaths [1]. In 2016 , the number of expected new cases is 26,370 in US, with 10,730 expected deaths [2]. The epidemic nature and high mortality of GC have driven researchers to assess the relative efficacy of various treatments. Surgery has been identified to be curative for early stage patients, and preoperative or postoperative chemotherapy and chemoradiotherapy can improve the remedy [3]. However, about $60 \%$ of the patients are diagnosed after 65 years old, when surgery is considered to be risky [4]. Thus, chemotherapy is widely used as a major treatment for its applicability to almost all patients.

Recently, it was reported that molecular targeted agents are able to significantly reinforce the efficacy of chemotherapy [5]. Several molecular targets and medications have been researched and combined with chemotherapy. One group of target molecules includes vascular endothelial growth factor (VEGF) and vascular endothelial factor receptor (VEGFR), both of which facilitates in the angiogenesis and metastasis of GC [6]. Another group of targets is related to epidermis. For instance, human epidermal growth factor receptor 2 (HER2) could stimulate the proliferation of tumor cells, and its overexpression triggers the development of cancer [7]. Epidermal growth factor receptor (EGFR) could also activate the macrophage in nonspecific immunity [8]. In 
addition, the mammalian target of rapamycin (mTOR) contributed to cellular apoptosis and proliferation [2]. Tyrosine kinase inhibitor (TKI) prohibits the expression of tyrosine kinase in order to stop the signal transduction for proteins [9] Hepatocyte growth factor receptor (HGFR) is encoded by c-MET gene and accelerates epithelialmesenchymal transition [2]. By using these target agents in chemotherapy, the efficacy could be multiplied.

Several reports have been published to illustrate the efficacy of molecular targeted treatment. A study in Japan reported that the use of Bevacizumab, an antibody of VEGF, could significantly increase progression-free survival (PFS). Ramucirumab, an antibody of VEGFR, was proved to be able to increase the overall survival (OS) rate in combination with paclitaxel [10]. TKI plus docetaxel would promote the overall response rate (ORR) compared to the monotherapy of docetaxel [11]. Although EGFR and mTOR targeted agents did not seem to significantly improve the effect of chemotherapy, these agents provided a new method for GC treatment. However, despite desirable results targeted therapies may bring, they were also associated with adverse events. According to the US National Cancer Institute (NCI), these alternative treatments may produce grade 3-4 adverse events [12]. Hence, in order to amplify the efficacy of chemotherapy while reduce severe adverse events as many as possible, this network meta-analysis (NMA) was designed to compare the relative efficacy and safety of different targeted agents in combination with chemotherapy and aimed to select an optimal targeted drug.

\section{RESULTS}

\section{Study characteristics}

As shown in Figure 1, through extensive searching and exclusion, 23 articles with 4,109 patients were finally obtained [1-3, 11, 13-31]. The baseline characteristics of all included studies shown presented in Table 1. All the 23 studies involved patients with advanced cancer, while some with metastatic cancer or unresectable caner. 12 of the studies were associated with first-line chemotherapy while 8 of them were associated with second-line chemotherapy. All the studies compared targeted agents with placebo (in combination with chemotherapy), with 11 for anti-VEGFR, 7 for anti-EGFR, 3 for anti-HER2, 2 included TKI, 1 for anti-mTOR and another one for antiMET (Figure 2).

\section{Survival outcomes}

Results of survival outcomes were shown in Table 2 and Figure 3. For one-year OS (1-OS), only VEFG(R) was superior to placebo $(\mathrm{HR}=0.77,95 \% \mathrm{CrI}=0.65-$ 0.91). While for one-year PFS (1-PFS), both HER2 and VEGF(R) were more effective than placebo $(\mathrm{HR}=0.75$,
$95 \% \mathrm{CrI}=0.56-1.00 ; \mathrm{HR}=0.67,95 \% \mathrm{CrI}=0.57-0.78$, respectively). In the meantime, $\operatorname{VEGF(R)}$ exhibited better performance than EGFR $(\mathrm{HR}=0.66,95 \% \mathrm{CrI}=$ $0.51-0.85)$. No significantly statistical difference was found in 2-OS, but in terms of 2-PFS, there was a big difference of efficacy among different drugs. Similar to 1-PFS, both HER2 and VEGF(R) were more effective than placebo $(\mathrm{HR}=0.76,95 \% \mathrm{CrI}=0.61-0.95 ; \mathrm{HR}=0.67$, $95 \% \mathrm{CrI}=0.60-0.76$, respectively), however, EGFR was the only one inferior to placebo $(\mathrm{HR}=1.22,95 \% \mathrm{CrI}=$ 1.03-1.45), and exhibited less satisfying results compared to HER2 ( $\mathrm{HR}=0.63,95 \% \mathrm{CrI}=0.47-0.82), \mathrm{m}-\mathrm{TOR}(\mathrm{HR}$ $=0.59,95 \% \mathrm{CrI}=0.40-0.88)$ and $\operatorname{VEGF}(\mathrm{R})(\mathrm{HR}=0.55$, $95 \% \mathrm{CrI}=0.45-0.68)$. Plus, VEGF(R) was more effective than MET (HR $=0.63,95 \% \mathrm{CrI}=0.42-0.95)$ and TKI (HR $=0.74,95 \% \mathrm{CrI}=0.56-0.99)$. Similar results repeated with respect to 3-OS, with EGFR inferior to placebo $(\mathrm{HR}=1.22,95 \% \mathrm{CrI}=1.02-1.44), \mathrm{HER} 2(\mathrm{HR}=1.50$, $95 \% \mathrm{CrI}=1.16-1.95)$ and $\operatorname{VEGF}(\mathrm{R})(\mathrm{HR}=1.40,95 \%$ $\mathrm{CrI}=1.14-1.73)$, while HER2 and $\operatorname{VEGF}(\mathrm{R})$ superior to placebo $(\mathrm{HR}=0.81,95 \% \mathrm{CrI}=0.67-0.98 ; \mathrm{HR}=0.87$, $95 \% \mathrm{CrI}=0.77-0.97)$.

\section{Response rate and adverse events (grade $\geq 3$ )}

Results of response rate and adverse events were exhibited in Table 3 and Figure 4. Consistent with the results with respect to survival, HER2 and $\operatorname{VEGF}(\mathrm{R})$ were the only two agents that brought higher response rate among patients compared with placebo $(\mathrm{OR}=2.03,95 \% \mathrm{CrI}=1.23-3.67 ; \mathrm{OR}=1.73,95 \% \mathrm{CrI}$ $=1.27-2.39$, respectively). As for adverse events, no significant statistical difference was detected in terms of vomiting, anaemia and nausea. M-TOR invited more thrombocytopenia events than EGFR (OR $=5.37$, $95 \% \mathrm{CrI}=1.11-37.71)$ and was associated with higher risk of neutropenia compared to placebo and EGFR $(\mathrm{OR}=12.81,95 \% \mathrm{CrI}=1.23-432.68 ; \mathrm{OR}=13.46,95 \%$ $\mathrm{CrI}=1.13-487.85$, respectively). $\mathrm{VEGF}(\mathrm{R})$ introduced more neutropenia events and fatigue events than placebo $(\mathrm{OR}=1.97,95 \% \mathrm{CrI}=1.21-3.22 ; \mathrm{OR}=1.90,95 \%$ $\mathrm{CrI}=1.22-2.72$ ), while TKI seemed to reduce the risk of fatigue for its superiority to all other drugs. In terms of diarrhea, EGFR, HER2 and VEGF(R) were associated with higher risk compared to placebo $(\mathrm{OR}=1.84,95 \%$ $\mathrm{CrI}=1.09-3.13 ; \mathrm{OR}=4.18,95 \% \mathrm{CrI}=2.41-7.61$; $\mathrm{OR}=1.99,95 \% \mathrm{CrI}=1.32-3.10$, respectively), and moreover, HER2 seemed to bring more diarrhea events than EGFR, TKI and VEGF(R) $(\mathrm{OR}=2.25,95 \%$ $\mathrm{CrI}=1.07-5.21 ; \mathrm{OR}=4.48,95 \% \mathrm{CrI}=1.11-17.46$; $\mathrm{OR}=2.08,95 \% \mathrm{CrI}=1.04-4.26$ ).

\section{Ranking of molecules of targeted therapy}

As was presented in Table 4, HER2 was the most effective drug with respect to OS while $\operatorname{VEGF}(\mathrm{R})$ invited 
Table 1: Main characteristics of included studies

\begin{tabular}{|c|c|c|c|c|c|c|c|c|c|c|}
\hline \multirow{2}{*}{ Study information } & \multirow{2}{*}{$\begin{array}{c}\text { Follow-up } \\
\text { (months) }\end{array}$} & \multirow{2}{*}{ Stage } & \multirow{2}{*}{ Chemotherapy } & \multicolumn{4}{|c|}{ Experiment group } & \multicolumn{2}{|c|}{ Placebo group } & \multirow{2}{*}{ Outcomes } \\
\hline & & & & Size & Age (range) & Target & Regimen & Size & Age (range) & \\
\hline Bang, 2010, Korea & 34 & Advanced & First-line & 294 & 59 & HER2 & Trastuzumab & 290 & 59 & (1)(2)(3)(4)(5)(6)(7)(8)(9)(10) \\
\hline Casak, 2015, USA & 30 & $\begin{array}{l}\text { Metastatic/ } \\
\text { unresectable }\end{array}$ & First-line & 327 & - & VEGF(R) & Ramucirumab & 329 & - & (1)(5)(6)(7)(8) \\
\hline Du, 2015, China & 60 & $\begin{array}{l}\text { Advanced/ } \\
\text { metastatic }\end{array}$ & First-line & 31 & $48(22-75)$ & EGFR & Nimotuzumab & 31 & $53(30-76)$ & (1)(2)(3)(4)(5)(6)(7)(8)(9)(10) \\
\hline Fuchs, 2014, USA & 28 & Advanced & First-line & 238 & $60(52-67)$ & VEGF(R) & Ramucirumab & 117 & $60(51-71)$ & (1)(2)(3)(5) 6)(7) \\
\hline Hecht, 2016, USA & 45 & $\begin{array}{l}\text { Advanced/ } \\
\text { metastatic }\end{array}$ & - & 249 & $61(19-86)$ & HER2 & Lapatinib & 238 & $59(27-84)$ & (1)(2)(3)(4)(5)(6)(7)(8)(9) \\
\hline Lordick, 2013, Germany & 42 & $\begin{array}{l}\text { Advanced/ } \\
\text { metastatic }\end{array}$ & First-line & 455 & $60(23-84)$ & EGFR & Cetuximab & 449 & $59(18-81)$ & (1)(2)(3)(4)(5)(6)(7)(8)(9)(10) \\
\hline Manish, 2016, Multicenter & 12 & Advanced & First-line & 62 & $59(33-78)$ & MET & Onartuzumab & 61 & $57(31-82)$ & (1)(2)(3)(9)(10) \\
\hline Markus, 2016, Germany & 12 & Advanced & Second-line & 45 & $62(37-76)$ & TKI & Sunitinib & 45 & $57(28-84)$ & (1)(2)(3)(4)(5)(6)(8)(9) \\
\hline \multirow[t]{2}{*}{ Muro, 2016, Japan } & 28 & Advanced & Second-line & 109 & $62(28-76)$ & VEGF(R) & Ramucirumab & 114 & $62(28-81)$ & (1)(2)(3)(4)(5) (6) (7) (8)(9)(10) \\
\hline & & & & 221 & $60(25-83)$ & VEGF(R) & Ramucirumab & 221 & $60(24-84)$ & (1)(2)(3)(4) (5) (6) (7) (8) (9)(10) \\
\hline Ohtsu, 2011, Japan & 24 & Advanced & First-line & 387 & $58(22-81)$ & $\operatorname{VEGF(R)}$ & Bevacizumab & 387 & $59(22-82)$ & (1)(2)(3)(4)(6)(7)(8)(9) \\
\hline Ohtsu, 2013, Japan & 24 & Advanced & Second-line & 439 & $62(20-86)$ & mTOR & Everolimus & 217 & $62(26-88)$ & (1)(2)(3)(4)(5)(6)(7)(8)(9)(10) \\
\hline Rao, 2010, UK & 24 & Advanced & First-line & 35 & $59(29-79)$ & EGFR & Matuzumab & 36 & $64(36-76)$ & (1)(2)(3)(4) (5) (6) (7) (8)(9)(10) \\
\hline Richards, 2013, USA & 36 & Advanced & First-line & 75 & $64(25-84)$ & EGFR & Cetuximab & 75 & $62(25-82)$ & (1)(2)(3)(5)(8)(9)(10) \\
\hline Satoh, 2015, Japan & 20 & Advanced & Second-line & 40 & 60 & EGFR & Nimotuzumab & 42 & 64 & (1)(2)(4)(5)(6)(7)(8)(9)(10) \\
\hline Satoh, 2014, Japan & 45 & Advanced & Second-line & 132 & $61(32-79)$ & HER2 & Lapatinib & 129 & $62(22-80)$ & (1)(2)(3)(4)(5)(6)(7)(8) \\
\hline Shen, 2015, China & 27 & $\begin{array}{l}\text { Advanced/ } \\
\text { metastatic }\end{array}$ & First-line & 100 & - & VEGF(R) & Bevacizumab & 102 & - & (1)(2)(3)(4)(6)(7)(8)(9)(10) \\
\hline \multirow[t]{2}{*}{ Shitara, 2016, Japan } & 28 & $\begin{array}{l}\text { Advanced/ } \\
\text { metastatic }\end{array}$ & Second-line & 68 & $64(34-76)$ & VEGF(R) & Ramucirumab & 72 & $64.5(29-76)$ & (1)(2)(3)(5)(6)(8)(9) \\
\hline & & /unresectable & & 198 & $60(25-83)$ & VEGF(R) & Ramucirumab & 200 & $61(24-84)$ & (1)(2)(3)(5)(6)(8)(9) \\
\hline Tebbutt, 2016, Australia & 18 & Advanced & First-line & 37 & $64(40-79)$ & EGFR & Panitumumab & 39 & $59(37-77)$ & (1)(2)(4)(5)(6)(8)(9) \\
\hline Wilke, 2014, Germany & 28 & Advanced & Second-line & 330 & $61(25-83)$ & VEGF(R) & Ramucirumab & 335 & $61(24-84)$ & (1)(2)(3)(4)(5)(6)(7)(8)(9)(10) \\
\hline $\mathrm{Xu}, 2013$, China & 30 & Advanced & - & 80 & - & VEGF(R) & Endostar & 85 & - & (2)(3)(4)(7)(8)(9)(10) \\
\hline Xu, 2014, China & 15 & Advanced & - & 17 & - & EGFR & Nimotuzumab & 17 & - & (1)(2)(3) \\
\hline Yi, 2012, Korea & 24 & Advanced & Second-line & 56 & $54(20-72)$ & TKI & Sunitinib & 49 & $52(36-70)$ & (1)(2)(3)(7)(8)(9)(10) \\
\hline Yoon, 2016, USA & 17 & Advanced & First-line & 84 & $65(27-83)$ & VEGF(R) & Ramucirumab & 84 & $64(34-82)$ & (1)(2) (3)(4)(5)(8)(9)(10) \\
\hline
\end{tabular}

most desirable results in terms of PFS, and both of them were associated with higher ORR. TKI turned out to be the most effective drug reducing the incidence of fatigue and diarrhoea, while EGFR was associated with less neutropenia events and thrombocytopenia compared with other treatments. $\operatorname{VEGF}(\mathrm{R})$ was effective in controlling the incidence of anaemia while $\mathrm{m}$-TOR was associated with the least risk of vomiting.

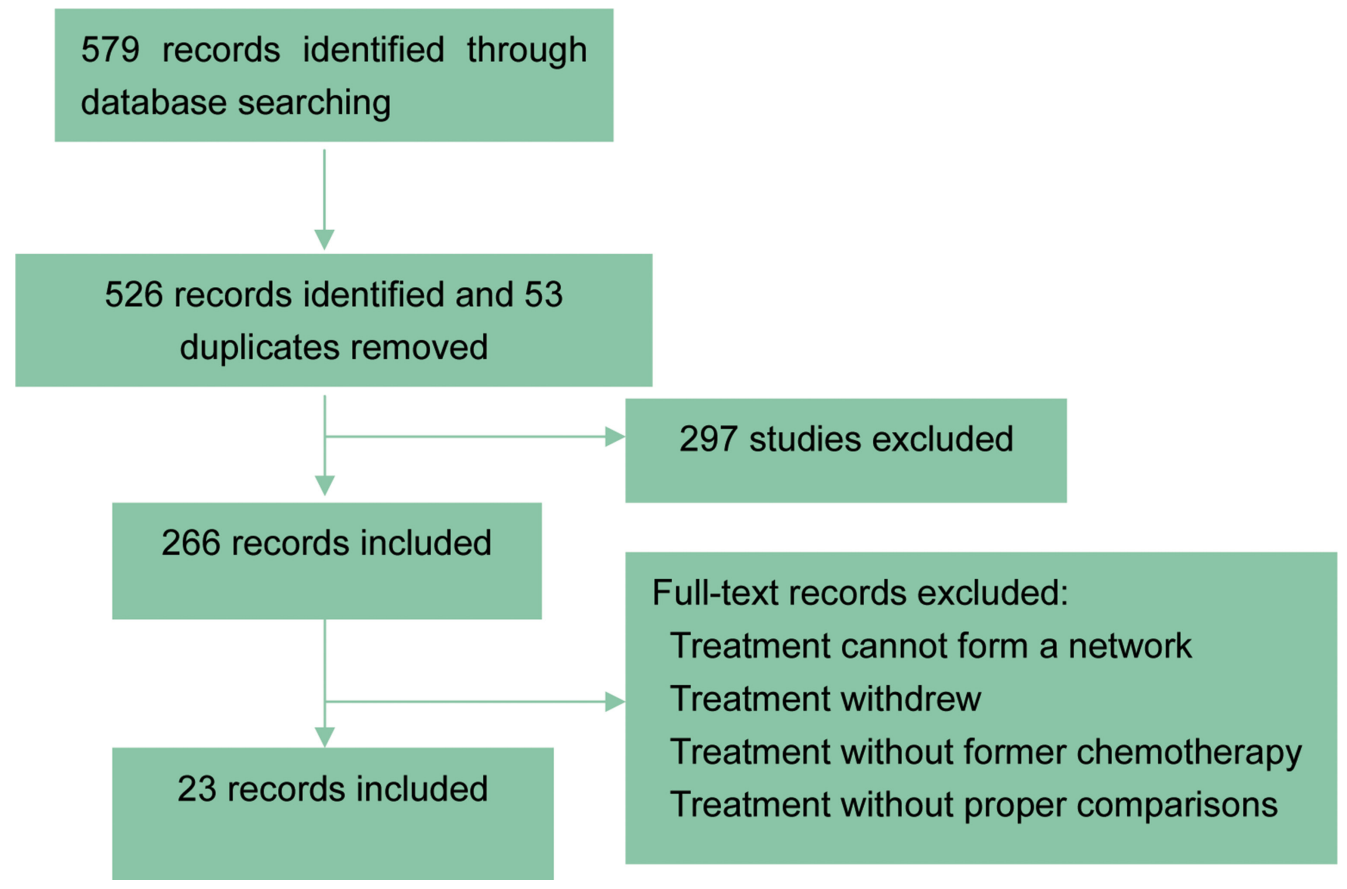

Figure 1: Flow charts of included studies. The searching and exclusion process was illustrated. 
Table 2: Network meta-analysis results of survival outcomes

\begin{tabular}{|c|c|c|c|c|c|c|c|c|}
\hline \multirow{7}{*}{ 1-OS } & Placebo & $1.01(0.83,1.22)$ & $0.75(0.56,1.00)$ & $1.08(0.65,1.80)$ & $0.72(0.44,1.18)$ & $0.92(0.64,1.30)$ & $0.67(0.57,0.78)$ & \multirow{7}{*}{ 1-PFS } \\
\hline & $0.98(0.79,1.21)$ & EGFR & $0.74(0.52,1.05)$ & $1.07(0.62,1.85)$ & $0.71(0.42,1.21)$ & $0.91(0.61,1.35)$ & $0.66(0.51,0.85)$ & \\
\hline & $0.76(0.55,1.05)$ & $0.78(0.53,1.14)$ & HER2 & $1.44(0.80,2.60)$ & $0.96(0.54,1.70)$ & $1.22(0.77,1.93)$ & $0.89(0.64,1.25)$ & \\
\hline & $0.97(0.55,1.71)$ & $0.99(0.54,1.81)$ & $1.27(0.66,2.44)$ & MET & $0.67(0.33,1.36)$ & $0.85(0.45,1.58)$ & $0.62(0.36,1.06)$ & \\
\hline & $0.87(0.51,1.48)$ & $0.89(0.50,1.57)$ & $1.14(0.62,2.12)$ & $0.90(0.41,1.95)$ & mTOR & $1.27(0.69,2.33)$ & $0.93(0.55,1.56)$ & \\
\hline & $0.82(0.56,1.21)$ & $0.84(0.54,1.30)$ & $1.08(0.66,1.78)$ & $0.85(0.43,1.68)$ & $0.95(0.49,1.82)$ & TKI & $0.73(0.49,1.08)$ & \\
\hline & $0.77(0.65,0.91)$ & $0.79(0.60,1.03)$ & $1.01(0.70,1.45)$ & $0.79(0.44,1.43)$ & $0.88(0.51,1.54)$ & $0.93(0.61,1.42)$ & VEGF (R) & \\
\hline \multirow{7}{*}{ 2-OS } & Placebo & $1.22(1.03,1.45)$ & $0.76(0.61,0.95)$ & $1.07(0.73,1.58)$ & $0.72(0.50,1.04)$ & $0.91(0.70,1.18)$ & $0.67(0.60,0.76)$ & \multirow{7}{*}{ 2-PFS } \\
\hline & $0.99(0.81,1.20)$ & EGFR & $0.63(0.47,0.82)$ & $0.88(0.57,1.34)$ & $0.59(0.40,0.88)$ & $0.74(0.54,1.02)$ & $0.55(0.45,0.68)$ & \\
\hline & $0.81(0.60,1.09)$ & $0.82(0.57,1.17)$ & HER2 & $1.40(0.90,2.18)$ & $0.94(0.62,1.44)$ & $1.19(0.85,1.68)$ & $0.88(0.69,1.13)$ & \\
\hline & $1.10(0.65,1.87)$ & $1.12(0.64,1.96)$ & $1.36(0.74,2.50)$ & MET & $0.67(0.40,1.14)$ & $0.85(0.53,1.36)$ & $0.63(0.42,0.95)$ & \\
\hline & $0.90(0.54,1.49)$ & $0.91(0.53,1.57)$ & $1.11(0.62,2.00)$ & $0.82(0.39,1.70)$ & mTOR & $1.26(0.81,1.97)$ & $0.94(0.64,1.37)$ & \\
\hline & $0.86(0.60,1.24)$ & $0.87(0.58,1.32)$ & $1.06(0.66,1.70)$ & $0.78(0.41,1.48)$ & $0.95(0.51,1.78)$ & TKI & $0.74(0.56,0.99)$ & \\
\hline & $0.86(0.73,1.01)$ & $0.87(0.68,1.13)$ & $1.06(0.76,1.49)$ & $0.78(0.45,1.36)$ & $0.96(0.56,1.63)$ & $1.00(0.67,1.49)$ & VEGF (R) & \\
\hline \multirow{7}{*}{ 3-OS } & Placebo & $1.08(0.81,1.46)$ & $0.77(0.58,1.02)$ & $1.08(0.59,1.98)$ & $0.66(0.41,1.06)$ & $0.91(0.59,1.41)$ & $0.66(0.56,0.77)$ & \multirow{7}{*}{ 3-PFS } \\
\hline & $1.22(1.02,1.44)$ & EGFR & $0.71(0.47,1.07)$ & $1.00(0.51,1.95)$ & $0.61(0.35,1.06)$ & $0.84(0.50,1.43)$ & $0.61(0.43,0.85)$ & \\
\hline & $0.81(0.67,0.98)$ & $0.67(0.51,0.86)$ & HER2 & $1.40(0.72,2.73)$ & $0.86(0.50,1.48)$ & $1.19(0.71,1.98)$ & $0.85(0.62,1.17)$ & \\
\hline & $1.06(0.59,1.90)$ & $0.87(0.47,1.60)$ & $1.31(0.71,2.42)$ & MET & $0.61(0.28,1.31)$ & $0.85(0.40,1.78)$ & $0.61(0.33,1.14)$ & \\
\hline & $0.90(0.64,1.28)$ & $0.74(0.50,1.09)$ & $1.11(0.74,1.66)$ & $0.85(0.43,1.68)$ & mTOR & $1.39(0.73,2.62)$ & $1.00(0.61,1.64)$ & \\
\hline & $0.88(0.59,1.31)$ & $0.72(0.47,1.11)$ & $1.09(0.70,1.69)$ & $0.83(0.41,1.68)$ & $0.98(0.58,1.66)$ & TKI & $0.72(0.45,1.14)$ & \\
\hline & $0.87(0.77,0.97)$ & $0.71(0.58,0.88)$ & $1.07(0.85,1.34)$ & $0.82(0.45,1.48)$ & $0.96(0.67,1.39)$ & $0.98(0.65,1.49)$ & VEGF (R) & \\
\hline
\end{tabular}

Abbreviations: HER2, Human epidermal growth factor receptor-2; EGFR, epidermal growth factor receptor; VEGF(R), vascular endothelial growth factor (receptor); TKI, tyrosine kinase inhibitor; mTOR, mammalian target of rapamycin; HGRF, Hepatocyte growth factor receptor.

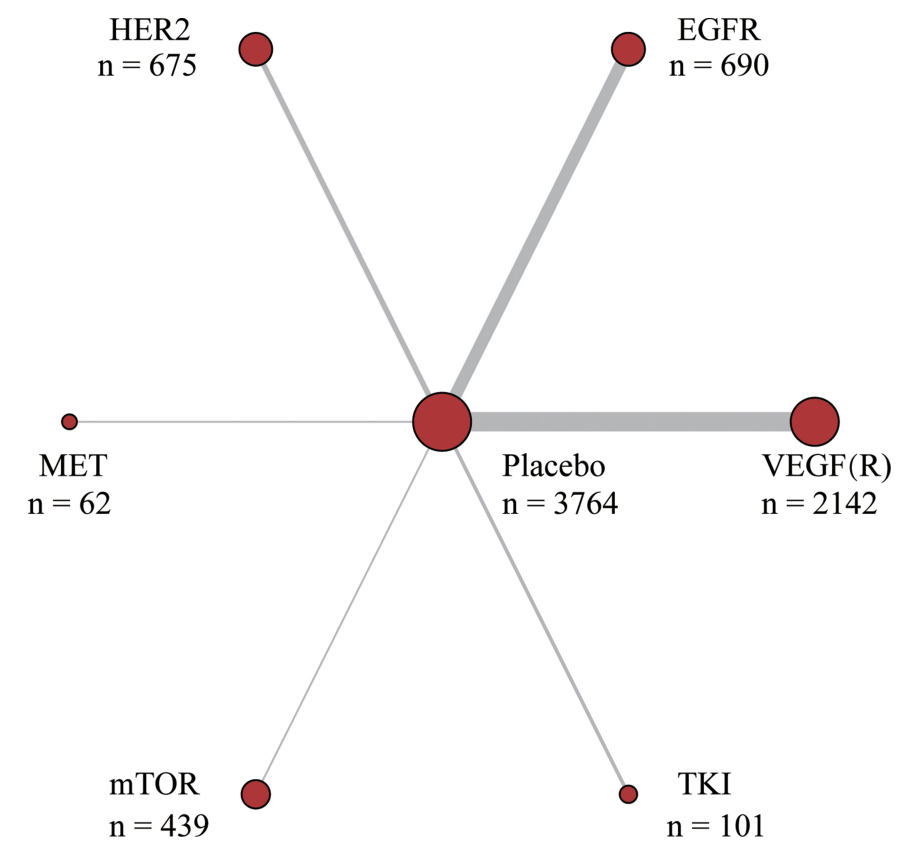

Figure 2: Network plot of molecular targeted agents for gastric cancer. The width of the lines is proportional to the number of trials comparing each pair of treatments target; the area of circles represents the cumulative number of patients for each intervention. 
Table 3: Network meta-analysis results of response rate and adverse events

\begin{tabular}{|c|c|c|c|c|c|c|c|c|}
\hline \multirow[t]{7}{*}{ ORR } & Placebo & $1.28(0.71,2.97)$ & $1.11(0.51,2.53)$ & $1.02(0.29,3.82)$ & $0.98(0.14,7.39)$ & $0.97(0.58,1.77)$ & - & Nausea \\
\hline & $1.11(0.68,1.88)$ & EGFR & $0.86(0.27,2.25)$ & $0.79(0.16,3.16)$ & $0.76(0.09,5.99)$ & $0.75(0.29,1.67)$ & - & \\
\hline & $2.03(1.23,3.67)$ & $1.84(0.90,3.90)$ & HER2 & $0.92(0.20,4.26)$ & $0.89(0.11,7.46)$ & $0.87(0.34,2.36)$ & - & \\
\hline & $2.29(0.63,10.38)$ & $2.08(0.51,9.97)$ & $1.12(0.27,5.47)$ & mTOR & $0.96(0.09,10.80)$ & $0.94(0.24,3.97)$ & - & \\
\hline & $1.68(0.70,4.06)$ & $1.54(0.54,4.10)$ & $0.83(0.29,2.25)$ & $0.73(0.13,3.53)$ & TKI & $1.00(0.13,7.77)$ & - & \\
\hline & $1.73(1.27,2.39)$ & $1.57(0.85,2.80)$ & $0.85(0.44,1.55)$ & $0.76(0.16,2.89)$ & $1.03(0.41,2.59)$ & VEGF (R) & - & \\
\hline & $1.20(0.41,3.56)$ & $1.08(0.33,3.53)$ & $0.58(0.17,1.93)$ & $0.52(0.08,2.86)$ & $0.71(0.18,2.83)$ & $0.69(0.23,2.14)$ & MET & \\
\hline \multirow[t]{7}{*}{ Neutropenia } & Placebo & $0.77(0.33,1.57)$ & $1.75(0.48,6.82)$ & $3.74(0.57,38.09)$ & $4.06(0.96,23.57)$ & $0.90(0.09,10.28)$ & $1.21(0.58,2.56)$ & Thrombocytopenia \\
\hline & $0.95(0.46,1.99)$ & EGFR & $2.29(0.54,11.94)$ & $5.00(0.63,59.15)$ & $5.37(1.11,37.71)$ & $1.20(0.11,15.80)$ & $1.58(0.57,4.95)$ & \\
\hline & $2.12(0.86,5.81)$ & $2.23(0.70,7.69)$ & HER2 & $2.14(0.21,31.19)$ & $2.34(0.32,20.91)$ & $0.51(0.03,7.85)$ & $0.68(0.14,3.10)$ & \\
\hline & $1.75(0.34,8.85)$ & $1.84(0.31,10.70)$ & $0.83(0.12,5.16)$ & MET & $1.08(0.07,14.01)$ & $0.23(0.01,5.58)$ & $0.32(0.03,2.44)$ & \\
\hline & $12.81(1.23,432.68)$ & $13.46(1.13,487.85)$ & $6.05(0.45,223.63)$ & $7.54(0.41,347.23)$ & mTOR & $0.21(0.01,3.67)$ & $0.30(0.04,1.49)$ & \\
\hline & $3.10(0.92,10.49)$ & $3.25(0.79,13.20)$ & $1.46(0.30,6.62)$ & $1.77(0.23,13.33)$ & $0.24(0.01,3.53)$ & TKI & $1.34(0.10,15.18)$ & \\
\hline & $1.97(1.21,3.22)$ & $2.08(0.85,4.95)$ & $0.94(0.30,2.59)$ & $1.13(0.21,6.17)$ & $0.15(0.00,1.73)$ & $0.64(0.17,2.36)$ & VEGF (R) & \\
\hline \multirow[t]{6}{*}{ Fatigue } & Placebo & $0.90(0.55,1.51)$ & $1.31(0.84,2.10)$ & $1.35(0.77,2.41)$ & $1.04(0.29,4.14)$ & $0.85(0.66,1.11)$ & & Anaemia \\
\hline & $1.43(0.84,2.39)$ & EGFR & $1.46(0.73,2.86)$ & $1.51(0.70,3.16)$ & $1.16(0.29,5.26)$ & $0.95(0.53,1.68)$ & & \\
\hline & $1.77(0.90,3.78)$ & $1.22(0.53,3.16)$ & HER2 & $1.04(0.50,2.16)$ & $0.79(0.20,3.46)$ & $0.65(0.38,1.09)$ & & \\
\hline & $1.62(0.61,4.39)$ & $1.13(0.38,3.49)$ & $0.91(0.26,3.03)$ & mTOR & $0.77(0.19,3.22)$ & $0.63(0.34,1.16)$ & & \\
\hline & $0.13(0.01,1.19)$ & $0.09(0.00,0.86)$ & $0.07(0.00,0.77)$ & $0.08(0.00,0.96)$ & TKI & $0.83(0.20,3.06)$ & & \\
\hline & $1.90(1.22,2.72)$ & $1.32(0.67,2.48)$ & $1.07(0.43,2.29)$ & $1.19(0.38,3.16)$ & $14.44(1.51,333.62)$ & VEGF (R) & & \\
\hline \multirow[t]{6}{*}{ Vomiting } & Placebo & $1.84(1.09,3.13)$ & $4.18(2.41,7.61)$ & $4.53(0.96,40.45)$ & $0.92(0.29,3.39)$ & $1.99(1.32,3.10)$ & & Diarrhoea \\
\hline & $1.04(0.57,1.99)$ & EGFR & $2.25(1.07,5.21)$ & $2.44(0.47,22.87)$ & $0.51(0.14,1.97)$ & $1.08(0.57,2.16)$ & & \\
\hline & $1.04(0.56,1.93)$ & $1.00(0.41,2.36)$ & HER2 & $1.08(0.20,10.59)$ & $0.22(0.06,0.90)$ & $0.48(0.23,0.96)$ & & \\
\hline & $0.72(0.24,2.16)$ & $0.69(0.19,2.39)$ & $0.69(0.20,2.41)$ & mTOR & $0.20(0.02,1.51)$ & $0.44(0.05,2.23)$ & & \\
\hline & $0.93(0.15,6.11)$ & $0.90(0.13,6.30)$ & $0.89(0.13,6.17)$ & $1.32(0.15,11.13)$ & TKI & $2.14(0.57,7.77)$ & & \\
\hline & $0.90(0.61,1.31)$ & $0.86(0.40,1.75)$ & $0.86(0.41,1.77)$ & $1.25(0.39,3.94)$ & $0.94(0.15,6.17)$ & VEGF (R) & & \\
\hline
\end{tabular}

Abbreviations: HER2, Human epidermal growth factor receptor-2; EGFR, epidermal growth factor receptor; VEGF(R), vascular endothelial growth factor (receptor); TKI, tyrosine kinase inhibitor; mTOR, mammalian target of rapamycin; HGRF, Hepatocyte growth factor receptor

\section{Subgroup analysis}

The results of subgroup one, which associated with first-line chemotherapy, were presented in Table 3, Supplementary Table 1 and Supplementary Figures 1-2, while those of subgroup two, which associate with secondline chemotherapy were exhibited in Table 3, Supplementary Table 2 and Supplementary Figures 3-4. In general, the results of subgroup analysis were consistent with previous results. However, HER2 used in combination with secondline chemotherapy did not seem to function as well as that with that in first-line chemotherapy, according to SUCRA.

\section{Publication bias}

The estimate of publication bias was performed by the symmetry characteristics of the dots representing included trials with different colors in the funnel plots.
According to Supplementary Figure 5, all of the funnel plots were focused in the triangle funnel areas in left and right directions, which suggested that the distribution of dots verified no significant publication bias or small study effect among ORR and all adverse events.

\section{DISCUSSION}

In the present study, an NMA was performed to evaluate the safety and efficacy of different molecular targeted therapies including VEGF(R), EGFR, HER2, mTOR and TKI in combination with chemotherapy for patient with GC.

According to the results of this NMA, therapies targeting HER2 or VEGF(R) outperformed others with respect to survival outcomes and response rate, which was supported by previous studies A study conducted by Badiani et al. reported that patients treated with the above 

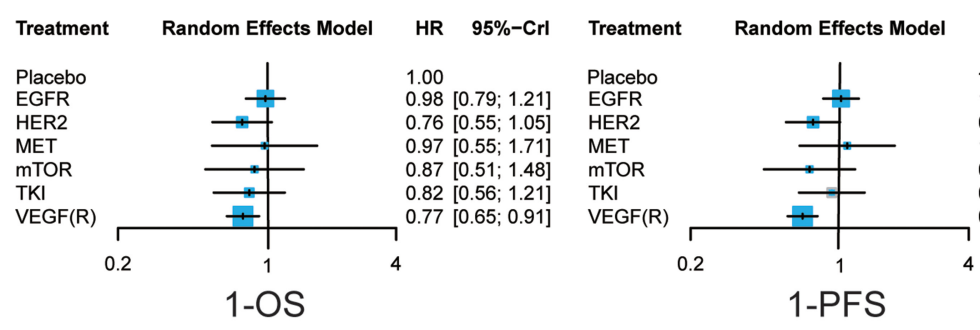

HR $\quad 95 \%-C r l$
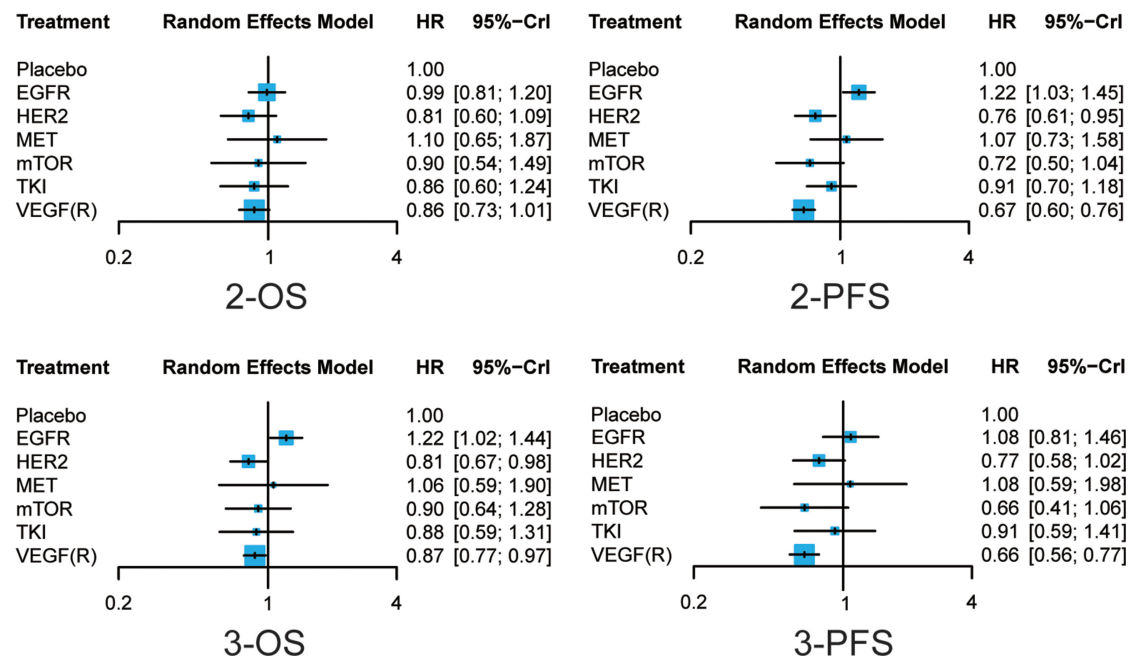

Figure 3: Forest plots of survival outcomes of different treatments Hazard ratios (HRs) with corresponding $95 \%$ credible intervals $(95 \% \mathrm{CrI})$ were used to measure the relative efficacy of different treatments.

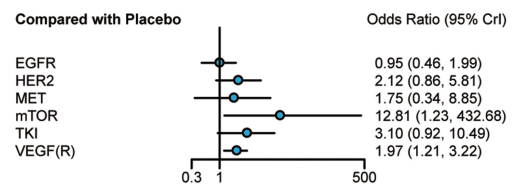

Neutropenia

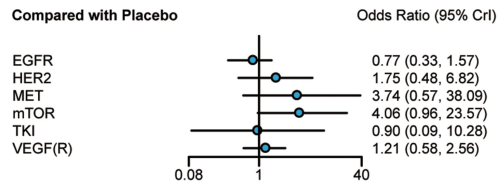

Thrombocytopenia

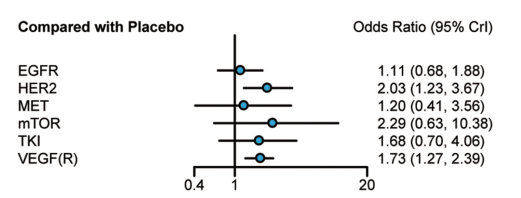

ORR

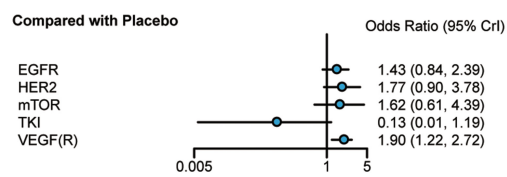

Fatigue
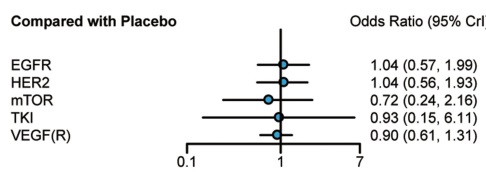

Vomiting

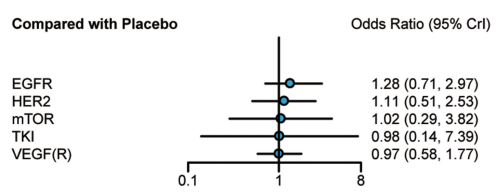

Nausea

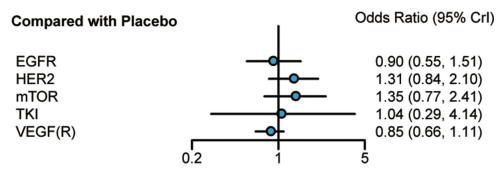

Anaemia
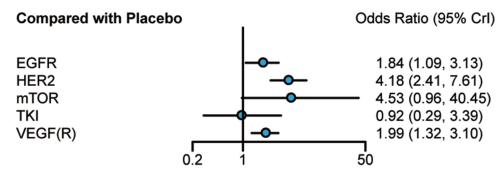

Diarrhoea

Figure 4: Forest plots of overall response rate and adverse events of different treatments. Odds ratios (ORs) with corresponding $95 \%$ credible intervals were used to measure the relative efficacy and safety of different treatments. 
Table 4: Surface under the cumulative rank curves value of different groups

\begin{tabular}{|c|c|c|c|c|c|c|c|c|c|c|c|c|c|c|}
\hline & 1-OS & 2-OS & 3-OS & 1-PFS & 2-PFS & 3-PFS & ORR & Fatigue & Vomiting & Diarrhoea & Nausea & Anaemia & Thrombocytopenia & Neutropenia \\
\hline \multicolumn{15}{|l|}{ All } \\
\hline Placebo & 0.231 & 0.290 & 0.356 & 0.265 & 0.318 & 0.287 & 0.151 & 0.750 & 0.424 & 0.881 & 0.571 & 0.538 & 0.683 & 0.851 \\
\hline EGFR & 0.295 & 0.354 & 0.080 & 0.252 & 0.053 & 0.191 & 0.261 & 0.441 & 0.400 & 0.522 & 0.315 & 0.684 & 0.826 & 0.852 \\
\hline vHER2 & 0.737 & 0.751 & 0.808 & 0.721 & 0.720 & 0.659 & 0.765 & 0.278 & 0.398 & 0.117 & 0.460 & 0.240 & 0.409 & 0.433 \\
\hline MET & 0.369 & 0.264 & 0.361 & 0.229 & 0.250 & 0.260 & 0.356 & - & - & - & - & - & 0.208 & 0.542 \\
\hline mTOR & 0.510 & 0.538 & 0.589 & 0.732 & 0.784 & 0.811 & 0.732 & 0.353 & 0.692 & 0.176 & 0.524 & 0.232 & 0.153 & 0.074 \\
\hline TKI & 0.605 & 0.628 & 0.619 & 0.424 & 0.469 & 0.427 & 0.594 & 0.980 & 0.499 & 0.838 & 0.535 & 0.512 & 0.657 & 0.293 \\
\hline VEGF(R) & 0.752 & 0.675 & 0.687 & 0.877 & 0.906 & 0.866 & 0.640 & 0.197 & 0.588 & 0.466 & 0.594 & 0.795 & 0.566 & $\mathrm{rt} 0.456$ \\
\hline \multicolumn{15}{|l|}{ Subgroup 1} \\
\hline Placebo & 0.361 & 0.463 & 0.451 & 0.416 & 0.415 & 0.378 & 0.297 & 0.870 & 0.452 & 0.976 & 0.668 & 0.353 & 0.748 & 0.548 \\
\hline EGFR & 0.028 & 0.101 & 0.108 & 0.079 & 0.083 & 0.160 & 0.269 & 0.359 & 0.431 & 0.430 & 0.350 & 0.695 & 0.831 & 0.674 \\
\hline HER2 & 0.924 & 0.929 & 0.892 & 0.908 & 0.904 & 0.991 & 0.823 & 0.321 & 0.641 & 0.168 & 0.570 & 0.176 & 0.421 & 0.645 \\
\hline MET & 0.430 & 0.308 & 0.399 & 0.288 & 0.323 & 0.244 & 0.501 & 0.207 & 0.225 & - & - & - & - & - \\
\hline VEGF(R) & 0.757 & 0.699 & 0.650 & 0.810 & 0.776 & 0.727 & 0.609 & 0.450 & 0.477 & 0.425 & 0.412 & 0.775 & 0.293 & 0.408 \\
\hline \multicolumn{15}{|l|}{ Subgroup 2} \\
\hline Placebo & 0.037 & 0.055 & 0.175 & 0.032 & 0.006 & 0.098 & 0.063 & 0.671 & 0.443 & 0.792 & 0.550 & 0.653 & 0.481 & 0.932 \\
\hline EGFR & 0.353 & 0.414 & 0.340 & 0.320 & - & - & - & 0.585 & 0.270 & 0.565 & 0.305 & 0.288 & 0.823 & 0.799 \\
\hline HER2 & 0.740 & 0.609 & 0.666 & 0.558 & 0.470 & 0.392 & 0.846 & 0.076 & 0.476 & 0.129 & 0.294 & 0.318 & - & 0.421 \\
\hline mTOR & 0.409 & 0.450 & 0.500 & 0.781 & 0.738 & 0.794 & 0.594 & 0.438 & 0.672 & 0.303 & 0.547 & 0.400 & 0.070 & 0.071 \\
\hline TKI & 0.527 & 0.572 & 0.546 & 0.311 & 0.289 & 0.295 & 0.407 & 0.939 & 0.476 & 0.766 & 0.559 & 0.579 & 0.540 & 0.415 \\
\hline VEGF(R) & 0.936 & 0.901 & 0.774 & 0.999 & 0.997 & 0.921 & 0.589 & 0.291 & 0.663 & 0.444 & 0.746 & 0.763 & 0.586 & 0.362 \\
\hline
\end{tabular}

Abbreviations: HER2, Human epidermal growth factor receptor-2; EGFR, epidermal growth factor receptor; VEGF(R), vascular endothelial growth factor (receptor); TKI, tyrosine kinase inhibitor; mTOR, mammalian target of rapamycin; HGRF, Hepatocyte growth factor receptor.

therapies exhibited significantly longer PFS, increased likelihood of disease control and enhanced ORR [32]. However, according our results, these two drugs were associated with higher risk of fatigue and diarrhea, which was also confirmed in other trials. For example, a study conducted by Bang et al. reported that HER2 invited more diarrhea and fatigue events [13], and another study designed by Fuchs et al. proved that VEGFR led to similar results [20]. Therefore, treatment recommendation should be made with caution.

Apart from VEGF(R) and HER2, agents targeting $\mathrm{m}$-TOR also yielded desirable results in terms of survival and response rate, and similarly, it may lead to serious adverse events such as neutropenia and diarrhea. However, the role of m-TOR may need to be justified further since only one of the included studies covered the analysis of $\mathrm{m}-\mathrm{TOR}$, and the same with MET.

Although conducted as meticulously as possible, this NMA still has some limitations. First of all, it is inevitable that the reliability this study may be diluted by heterogeneity. For example, different chemotherapy treatments may results in different survival outcomes and adverse events. A study conducted by Koizumi et al. reported that the use of S-1 plus cisplatin yielded more satisfying results with respect to OS [8]. This is probably why the performance of HER2 in second-line chemotherapy was not as desirable as that in first-line chemotherapy. Secondly, we did not conduct a stratified or subgroup analysis due to the shortage of evidence. Some included studies did not report any information about tumor stage, type or specific dosage in the chemotherapy, which may weaken the reliability of corresponding results and conclusions. On top of that, we discovered that some of the eligible studies did not implement any blinding procedures. Thus, the quality of studies may be suspected and the overall effect size may be biased. Finally, our study did not include any approach to assessing the potential publication bias and we did not include any unpublished studies or articles in which the effect size may be different from that in ours.

However, despite all the limitations, our study contained several strengths with respect to study design 
and evidence synthesize. Unlike other meta-analysis or NMA that usually contains literatures in the last two decades, all of the included studies were carried out between 2010 and 2016, which contributed to the reliability and clinical significance of this article. A typical issue often arises from the fact that the control of confounding factors is lost when evidence is synthesized from individual studies. For instance, age is usually a significant risk factor in epidemiology. If confounding factors such as age are not controlled, the association between other risk factors and diseases may be significantly altered. The median age of GC patients in our study did not appear to be distributed in a wide range and this phenomenon may also reduce the degree of heterogeneity. Another major distinctive feature of our study came from the approach when evidence was synthesized. Unlike other meta-analysis or NMA, in which evidence was synthesized by the specific treatments, our study combined evidence based on a specific pathway of each target agents. This novel approach helped us determine which pathway is appropriate to be targeted in order to enhance the efficacy or safety of chemotherapy.

In summary, $\operatorname{VEGF}(\mathrm{R})$ and HER2 were the best two targeted therapies for $\mathrm{GC}$, due to their high performance of efficacy outcomes whereas their adverse events should also be paid more attention. M-TOR may serve as alternative choice for its good performance with respect to survival outcomes, however, due to the lack of evidence, its role need to be further identified.

\section{MATERIALS AND METHODS}

\section{Search strategy}

We conducted the literature retrieval in PubMed, Embase and Cochrane Library systematically, aiming to select eligible articles which were designed as randomized controlled trials (RCTs) in GC patients. Following terms were used in the searching procedure: "vascular endothelial growth factor, vascular endothelial factor receptor, epidermal growth factor receptor, human epidermal growth factor receptor-2, mammalian target of rapamycin, tyrosine kinase inhibitor, chemotherapy, randomized controlled trials and gastric cancer". The searching procedure was accomplished by two investigators independently. Reviews and duplicated studies were removed after scanning the titles and abstracts.

\section{Inclusion criteria}

The following inclusion criteria were adopted: (1) studies must be RCTs; (2) targeted agents should be used in combination with chemotherapy ; (3) patients should be diagnosed with GC; (4) studies should cover at least one of the included outcomes The articles were excluded according to the following rules: (1) treatments cannot form a network; (2) treatment withdrew; (3) treatment without former chemotherapy; Once the included study list was finalized, the Jadad Scale was used to assess the quality of included studies (Supplementary Table 3) [33].

\section{Data extraction}

Two investigators independently searched the relevant data from eligible articles. In order to enhance the reliability and accuracy, a third party was involved to solve discrepancy. The following information is extracted: author, publication year, country, sample size, median age, target, treatments and outcomes, including OS and PFS of 1 year, 2 years and 3 years, ORR and 7 adverse events Among them, 1-year and 2-year survival were defined as short-term survival outcomes while 3-year and 5-year survival were long-term outcomes. Both survival outcomes and ORR were used to measure the relative efficacy of different target agents, while adverse events were used to measure their safety.

\section{Statistical analysis}

In this NMA, the odds rates (ORs) with corresponding 95\% credible intervals (CrIs) were utilized to measure response outcomes and adverse events. The hazard ratios (HRs) with 95\% CrIs were used to assess the long-term prognostic factors outcomes. Furthermore, surface under the cumulative ranking curve (SUCRA) was calculated to illustrate the ranking probability of each treatment under different endpoint, with higher values indicating better efficacy or safety.

After the analysis of all trials, included studies were divided into two subgroups according to the chemotherapy, with subgroup one referring to first-line chemotherapy while subgroup two referring to secondline chemotherapy. These two subgroups were analyzed followed the methods above.

\section{Abbreviations}

GC-Gastric cancer ; VEGF-vascular endothelial growth factor; VEGFR-vascular endothelial factor receptor; HER2-human epidermal growth factor receptor 2; EGFR-Epidermal growth factor receptor; mTORmammalian target of rapamycin; TKI-Tyrosine kinase inhibitor; OS-overall survival; BSC-best support care; ORR-overall response rate; AEs-adverse effects; GIgastrointestinal events; HE-hematological events; MAmeta-analyses; NMA-network meta-analysis; RCTsrandomized controlled trials

\section{Authors' contributions}

ZR, JPS and QXG Literature search, data extraction and manuscript writing; ZR and JPS: Statistical 
analysis; ZR, JPS and XFS: Manuscript revision and experimental design. HTH, KL and QXG are responsible for the overall content as the guarantor. All authors have read and approved the final manuscript.

\section{CONFLICTS OF INTEREST}

All authors declare no compete interests.

\section{FUNDING}

None.

\section{REFERENCES}

1. Yoon HH, Bendell JC, Braiteh FS, Firdaus I, Philip PA, Cohn AL, Lewis N, Anderson DM, Arrowsmith E, Schwartz JD, Gao L, Hsu Y, Xu Y, et al. Ramucirumab combined with FOLFOX as front-line therapy for advanced esophageal, gastroesophageal junction, or gastric adenocarcinoma: a randomized, double-blind, multicenter Phase II trial. Ann Oncol. 2016; 27:2196-2203.

2. Shah MA, Cho JY, Tan IB, Tebbutt NC, Yen CJ, Kang A, Shames DS, Bu L, Kang YK. A Randomized Phase II Study of FOLFOX With or Without the MET Inhibitor Onartuzumab in Advanced Adenocarcinoma of the Stomach and Gastroesophageal Junction. Oncologist. 2016; 21: 1085-1090.

3. Moehler M, Gepfner-Tuma I, Maderer A, ThussPatience PC, Ruessel J, Hegewisch-Becker S, Wilke H, Al-Batran SE, Rafiyan MR, Weissinger F, Schmoll HJ, Kullmann F, von Weikersthal LF, et al. Sunitinib added to FOLFIRI versus FOLFIRI in patients with chemorefractory advanced adenocarcinoma of the stomach or lower esophagus: a randomized, placebo-controlled phase II AIO trial with serum biomarker program. BMC Cancer. 2016; 16:699.

4. Li J, Qin S, Xu J, Xiong J, Wu C, Bai Y, Liu W, Tong J, Liu Y, Xu R, Wang Z, Wang Q, Ouyang X, et al. Randomized, Double-Blind, Placebo-Controlled Phase III Trial of Apatinib in Patients With Chemotherapy-Refractory Advanced or Metastatic Adenocarcinoma of the Stomach or Gastroesophageal Junction. J Clin Oncol. 2016; 34:1448-1454.

5. Hecht JR, Bang YJ, Qin SK, Chung HC, Xu JM, Park JO, Jeziorski K, Shparyk Y, Hoff PM, Sobrero A, Salman P, Li J, Protsenko SA, et al. Lapatinib in Combination With Capecitabine Plus Oxaliplatin in Human Epidermal Growth Factor Receptor 2-Positive Advanced or Metastatic Gastric, Esophageal, or Gastroesophageal Adenocarcinoma: TRIO013/LOGiC — A Randomized Phase III Trial. J Clin Oncol. 2016; 34:443-451.

6. Van Cutsem E, Boni C, Tabernero J, Massuti B, Middleton G, Dane F, Reichardt P, Pimentel FL, Cohn A, Follana P, Clemens M, Zaniboni A, Moiseyenko V, et al. Docetaxel plus oxaliplatin with or without fluorouracil or capecitabine in metastatic or locally recurrent gastric cancer: a randomized phase II study. Ann Oncol. 2015; 26:149-156.

7. Kataoka K, Tokunaga M, Mizusawa J, Machida N, Katayama H, Shitara K, Tomita T, Nakamura K, Boku N, Sano T, Terashima M, Sasako M, Stomach Cancer Study Group/Japan Clinical Oncology G. A randomized Phase II trial of systemic chemotherapy with and without trastuzumab followed by surgery in HER2positive advanced gastric or esophagogastric junction adenocarcinoma with extensive lymph node metastasis: Japan Clinical Oncology Group study JCOG1301 (Trigger Study). Jpn J Clin Oncol. 2015; 45:1082-1086.

8. Koizumi W, Narahara H, Hara T, Takagane A, Akiya T, Takagi M, Miyashita K, Nishizaki T, Kobayashi O, Takiyama W, Toh Y, Nagaie T, Takagi S, et al. S-1 plus cisplatin versus S-1 alone for first-line treatment of advanced gastric cancer (SPIRITS trial): a phase III trial. Lancet Oncol. 2008; 9:215-221.

9. Workman P, Brunton VG, Robins DJ. Tyrosine kinase inhibitors. Semin Cancer Biol. 1992; 3:369-381.

10. Wilke H, Muro K, Van Cutsem E, Oh SC, Bodoky G, Shimada Y, Hironaka S, Sugimoto N, Lipatov O, Kim TY, Cunningham D, Rougier P, Komatsu Y, et al. Ramucirumab plus paclitaxel versus placebo plus paclitaxel in patients with previously treated advanced gastric or gastrooesophageal junction adenocarcinoma (RAINBOW): a double-blind, randomised phase 3 trial. Lancet Oncol. 2014; 15:1224-1235.

11. Yi JH, Lee J, Lee J, Park SH, Park JO, Yim DS, Park YS, Lim HY, Kang WK. Randomised phase II trial of docetaxel and sunitinib in patients with metastatic gastric cancer who were previously treated with fluoropyrimidine and platinum. Br J Cancer. 2012; 106:1469-1474.

12. Zou K, Yang S, Zheng L, Yang C, Xiong B. Efficacy and safety of target combined chemotherapy in advanced gastric cancer: a meta-analysis and system review. BMC Cancer. 2016; $16: 737$.

13. Bang YJ, Van Cutsem E, Feyereislova A, Chung HC, Shen L, Sawaki A, Lordick F, Ohtsu A, Omuro Y, Satoh T, Aprile G, Kulikov E, Hill J, et al. Trastuzumab in combination with chemotherapy versus chemotherapy alone for treatment of HER2-positive advanced gastric or gastro-oesophageal junction cancer (ToGA): A phase 3, open-label, randomised controlled trial. The Lancet. 2010; 376:687-697.

14. Rao S, Starling N, Cunningham D, Sumpter K, Gilligan D, Ruhstaller T, Valladaresayerbes M, Wilke H, Archer C, Kurek R. Matuzumab plus epirubicin, cisplatin and capecitabine (ECX) compared with epirubicin, cisplatin and capecitabine alone as first-line treatment in patients with advanced oesophago-gastric cancer: a randomised, multicentre open-label phase II study. Annals of Oncology. 2010; 21:2213-2219.

15. Ohtsu A, Shah MA, Van Cutsem E, Rha SY, Sawaki A, Park SR, Lim HY, Yamada Y, Wu J, Langer B, 
Starnawski M, Kang YK. Bevacizumab in combination with chemotherapy as first-line therapy in advanced gastric cancer: A randomized, double-blind, placebo-controlled phase III study. Journal of Clinical Oncology. 2011; 29:3968-3976.

16. Lordick F, Kang YK, Chung HC, Salman P, Oh SC, Bodoky G, Kurteva G, Volovat C, Moiseyenko VM, Gorbunova V, Park JO, Sawaki A, Celik I, et al. Capecitabine and cisplatin with or without cetuximab for patients with previously untreated advanced gastric cancer (EXPAND): a randomised, open-label phase 3 trial. Lancet Oncol. 2013; 14:490-499.

17. Ohtsu A, Ajani JA, Bai YX, Bang YJ, Chung HC, Pan HM, Sahmoud T, Shen L, Yeh KH, Chin K, Muro K, Kim YH, Ferry D, et al. Everolimus for previously treated advanced gastric cancer: results of the randomized, double-blind, phase III GRANITE-1 study. J Clin Oncol. 2013; 31:3935-3943.

18. Richards D, Kocs DM, Spira AI, David McCollum A, Diab S, Hecker LI, Cohn A, Zhan F, Asmar L. Results of docetaxel plus oxaliplatin (DOCOX) $+/-$ cetuximab in patients with metastatic gastric and/or gastroesophageal junction adenocarcinoma: results of a randomised Phase 2 study. Eur J Cancer. 2013; 49:2823-2831.

19. Xu R, Ma N, Wang F, Ma L, Chen R, Chen R, Kebinu M, Ma L, Han Z, Ayixiamu, Mayier M, Su P, Naman Y, et al. Results of a randomized and controlled clinical trial evaluating the efficacy and safety of combination therapy with Endostar and S-1 combined with oxaliplatin in advanced gastric cancer. OncoTargets and Therapy. 2013; 6:925-929.

20. Fuchs CS, Tomasek J, Yong CJ, Dumitru F, Passalacqua R, Goswami C, Safran H, dos Santos LV, Aprile G, Ferry DR, Melichar B, Tehfe M, Topuzov E, et al. Ramucirumab monotherapy for previously treated advanced gastric or gastro-oesophageal junction adenocarcinoma (REGARD): an international, randomised, multicentre, placebocontrolled, phase 3 trial. Lancet. 2014; 383:31-39.

21. Satoh T, Xu RH, Chung HC, Sun GP, Doi T, Xu JM, Tsuji A, Omuro Y, Li J, Wang JW, Miwa H, Qin SK, Chung IJ, et al. Lapatinib plus paclitaxel versus paclitaxel alone in the second-line treatment of HER2-amplified advanced gastric cancer in Asian populations: TyTAN--a randomized, phase III study. J Clin Oncol. 2014; 32:2039-2049.

22. Wilke H, Muro K, Van Cutsem E, Oh SC, Bodoky G, Shimada Y, Hironaka S, Sugimoto N, Lipatov O, Kim TY, Cunningham D, Rougier P, Komatsu Y, et al. Ramucirumab plus paclitaxel versus placebo plus paclitaxel in patients with previously treated advanced gastric or gastrooesophageal junction adenocarcinoma (RAINBOW): a double-blind, randomised phase 3 trial. Lancet Oncol. 2014; 15:1224-1235.

23. Xu CD. Clinical study of nimotuzumab combined with chemotherapy in the treatment of late stage gastric cancer. Asian Pac J Cancer Prev. 2014; 15:10273-10276.

24. Casak SJ, Fashoyin-Aje I, Lemery SJ, Zhang L, Jin R, Li H, Zhao L, Zhao H, Zhang H, Chen H, He K, Dougherty M,
Novak R, et al. FDA Approval Summary: Ramucirumab for Gastric Cancer. Clin Cancer Res. 2015; 21:3372-3376.

25. Du F, Zheng Z, Shi S, Jiang Z, Qu T, Yuan X, Sun Y, Song Y, Yang L, Zhao J, Wang J, Chi Y. S-1 and Cisplatin With or Without Nimotuzumab for Patients With Untreated Unresectable or Metastatic Gastric Cancer: A Randomized, Open-Label Phase 2 Trial. Medicine (Baltimore). 2015; 94:e958.

26. Satoh T, Lee KH, Rha SY, Sasaki Y, Park SH, Komatsu Y, Yasui H, Kim TY, Yamaguchi K, Fuse N, Yamada Y, Ura T, Kim SY, et al. Randomized phase II trial of nimotuzumab plus irinotecan versus irinotecan alone as second-line therapy for patients with advanced gastric cancer. Gastric Cancer. 2015; 18:824-832.

27. Shen L, Li J, Xu J, Pan H, Dai G, Qin S, Wang L, Wang J, Yang Z, Shu Y, Xu R, Chen L, Liu Y, et al. Bevacizumab plus capecitabine and cisplatin in Chinese patients with inoperable locally advanced or metastatic gastric or gastroesophageal junction cancer: randomized, doubleblind, phase III study (AVATAR study). Gastric Cancer. 2015; 18:168-176.

28. Hecht JR, Bang YJ, Qin SK, Chung HC, Xu JM, Park JO, Jeziorski K, Shparyk Y, Hoff PM, Sobrero A, Salman P, Li J, Protsenko SA, et al. Lapatinib in combination with capecitabine plus oxaliplatin in human epidermal growth factor receptor 2-positive advanced or metastatic gastric, esophageal, or gastroesophageal adenocarcinoma: TRIO013/LOGiC - A randomized phase III trial. Journal of Clinical Oncology. 2016; 34:443-451.

29. Muro K, Oh SC, Shimada Y, Lee KW, Yen CJ, Chao Y, Cho JY, Cheng R, Carlesi R, Chandrawansa K, Orlando M, Ohtsu A. Subgroup analysis of East Asians in RAINBOW: A phase 3 trial of ramucirumab plus paclitaxel for advanced gastric cancer. J Gastroenterol Hepatol. 2016; 31:581-589.

30. Shitara K, Muro K, Shimada Y, Hironaka S, Sugimoto N, Komatsu Y, Nishina T, Yamaguchi K, Segawa Y, Omuro Y, Tamura T, Doi T, Yukisawa S, et al. Subgroup analyses of the safety and efficacy of ramucirumab in Japanese and Western patients in RAINBOW: a randomized clinical trial in second-line treatment of gastric cancer. Gastric Cancer. 2016; 19:927-938.

31. Tebbutt NC, Price TJ, Ferraro DA, Wong N, Veillard AS, Hall M, Sjoquist KM, Pavlakis N, Strickland A, Varma SC, Cooray P, Young R, Underhill C, et al. Panitumumab added to docetaxel, cisplatin and fluoropyrimidine in oesophagogastric cancer: ATTAX3 phase II trial. Br J Cancer. 2016; 114:505-509.

32. Badiani B, Maratea D, Messori A. Second-line treatments for advanced gastric cancer: Interpreting outcomes by network meta-analysis. World J Clin Oncol. 2015; 6:73-79.

33. Clark HD, Wells GA, Huet C, McAlister FA, Salmi LR, Fergusson D, Laupacis A. Assessing the quality of randomized trials: reliability of the Jadad scale. Control Clin Trials. 1999; 20:448-452. 